\title{
Association between Marginally Low Birth Weight and Obesity-Related Outcomes and Indirect Effects via Attention-Deficit Hyperactivity Disorder and Abnormal Eating
}

\author{
Xiaotong Wei ${ }^{a}$ Jiajin $\mathrm{Hu}^{\mathrm{a}}$ Yang $\mathrm{Liu}^{\mathrm{a}}$ Yanan Ma ${ }^{\mathrm{b}}$ Deliang Wen ${ }^{\mathrm{a}}$ \\ anstitute of Health Sciences, China Medical University, Shenyang, China; bepartment of Epidemiology and Health \\ Statistics, School of Public Health, China Medical University, Shenyang, China
}

\section{Keywords}

Obesity · Metabolic syndrome · Birth weight · Attention-

deficit hyperactivity disorder - Eating behaviour

\begin{abstract}
Introduction: Evidence of the association between children born with marginally low birth weight (MLBW) and obesityrelated outcomes was controversial, and our study aimed to examine the role of attention-deficit hyperactivity disorder (ADHD) and/or abnormal eating in these associations. Methods: A retrospective cohort study consisting of 677 Chinese children was conducted. Obesity-related outcomes (body mass index [BMI], waist circumference [WC], skinfold thickness [SF], body fat, blood pressure, lipids, and blood glucose), behaviour problems (ADHD and eating behaviour) and birth weight were collected. Mediation analyses were used to explore whether ADHD and/or abnormal eating was an intermediary factor in the MLBW-OB relationship. Results: Children with MLBW tended to have higher SF, triglycerides, fasting blood glucose, waistline, body fat, and abdominal obesity risks. Birth weight was negatively related to obesityrelated outcomes, and the associations were mediated, partially, by the increased risk of ADHD or abnormal eating be-
\end{abstract}

karger@karger.com www.karger.com/ofa

Karger"

GOPEN ACCESS
(C) 2021 The Author(s)

Published by S. Karger AG, Basel

This is an Open Access article licensed under the Creative Commons Attribution-NonCommercial-4.0 International License (CC BY-NC) (http://www.karger.com/Services/OpenAccessLicense), applicable to the online version of the article only. Usage and distribution for commercial purposes requires written permission. haviour after adjustment for the BMI $Z$ score. Furthermore, lower birth weight predicted higher WC indirectly through emotional overeating caused by ADHD ( $\beta$ : $-0.10 ; 95 \%$ confidence interval: $-0.19,-0.01)$. Conclusion: Our study suggests the hypothetical role of ADHD and abnormal eating as underlying mechanisms in the association between MLBW and obesity-related outcomes, providing novel scientific evidence for childhood development interventions.

(C) 2021 The Author(s)

Published by S. Karger AG, Basel

\section{Introduction}

Childhood obesity and metabolic syndrome have become significant global public health challenges, with over 42 million children under the age of 5 years threatened by obesity [1]. In China, overweight and obesity rates reached $16.9 \%$ in 2015 (up from $7.4 \%$ in 1985) and have remained stable to date [2]. Low birth weight (LBW) could be one key indicator of adverse reactions to foetal life and has been associated with obesity and its related outcomes [3-5]. The developmental origin of health and disease hypothesis proposes that an inadequate prenatal environment during foetal life could lead to permanent 
changes in body composition and metabolic parameters that persist into adulthood $[6,7]$. The relationships between birth weight and anthropometric measurements, such as waist circumference (WC), fat mass, and levels of fat or glucose in the blood, have been demonstrated, and a lower birth weight has been related to more significant central obesity and a higher percentage of fat mass or levels of lipids [8-10]. However, the results, especially in marginally low birth weight (MLBW, 2,000-2,500 g) children, have been few and inconsistent [11]. The majority of children with LBW are born with MLBW, and the prevalence of overweight and obesity in this group has been less explored, especially in healthy children. However, just one recent study found no significant relationship between MLBW and obesity and body composition [12], so further research is needed.

Furthermore, it is still unclear how comorbid factors could be used as mediators in the pathway linking birth weight to obesity-related outcomes. Attention-deficit hyperactivity disorder (ADHD) has hardly been studied as an intermediary in this correlation between LBW and obesity. Although results were mixed across studies, meta-analysis research showed significant relationships between LBW and ADHD or ADHD and obesity-related outcomes. Concerning the LBW-ADHD relationship, one recent meta-analysis concluded that LBW subjects had a higher ADHD risk (OR: 3.04; 95\% confidence interval [CI]: 2.20-4.20) [13], but in the Generation $\mathrm{R}$ Study, the children with LBW were not associated with attention problem scores after adjusting for confounders [14]. In addition, mounting evidence suggested that ADHD had a unique relationship with obesity [15], but less is known about associations of ADHD with obesityrelated outcomes. Given that LBW is a risk factor for obesity-related outcomes and that both children with obesity-related outcomes and those born with LBW had a higher prevalence of hyperkinetic syndrome than in controls, it is plausible that ADHD symptoms, at least partially, mediate the relationship between birth weight and obesity-related outcomes. Following this reasoning, a recent study using a sample of 615 children aged $6-18$ years found that overweight was observed significantly more often in boys with ADHD, and after adjusting for birth weight, this relationship was attenuated [16]. It is of note that this study only analysed the situation of overweight and obesity in boys. Therefore, it is necessary to explore further the mediating role of ADHD in this relationship between LBW and other obesity-related outcomes.

The mechanisms, which may explain the link between LBW, ADHD, and obesity, involve the dopaminergic sys- tem and behavioural effects of hyperactivity and inattention, leading to weight gain through abnormal eating [17]. To better understand the underlying mechanisms of comorbidity, eating behaviour should be considered as a critical mediating factor. LBW affects neural development, such as central nervous system appetite control and the desire for specific foods [18]. Furthermore, the mothers of those newborns with lower birth weight may be more likely to feed them more foods to encourage their child to grow larger, leading to childhood obesity or the formation of poor eating habits, especially in satiety and emotional eating [19]. On the other hand, those diagnosed with ADHD often experience dysregulation of dopamine and impairment in self-regulating function, which further as a result of disordered eating patterns [20]. A large clinical study found a significant relationship between eating behaviour and ADHD [21]. Nevertheless, there are currently no mature studies that have assessed these relationships by multiple-step multiplemediator models.

In this study, we used the simple mediation model and multiple-step multiple-mediator models to explore whether associations between MLBW and obesity-related outcomes were mediated by ADHD and/or eating behaviour. As shown in Figure 1, mediation hypotheses posit how, or by what means, an independent variable (X) affects a dependent variable $(\mathrm{Y})$ through one or more potential mediators (M) [22]. Moreover, our study used bootstrapping, a non-parametric resampling procedure, to test hypotheses about mediation. Compared with conventional methods, bootstrapping has higher power while maintaining reasonable control over the type I error rate [23].

To our knowledge, this is one of the first studies to explore the relationships between MLBW, ADHD, eating behaviour, and obesity-related outcomes, which if addressed would enhance the understanding of potential associations and provide evidence for public health interventions aimed at reducing childhood obesity and its related comorbidities. Thus, our study had three aims. Firstly, we sought to explore whether ADHD was an intermediary factor in the relationship between birth weight and obesity-related outcomes (Fig. 1a). Secondly, we intended to evaluate whether eating behaviour was an intermediary factor in the relationship between birth weight and obesity-related outcomes (Fig. 1b). Finally, our third aim was to assess whether eating behaviour is part of the foundation for the link between MLBW and the risks of child obesity-related outcomes in the context of deficits in ADHD (Fig. 1c). 


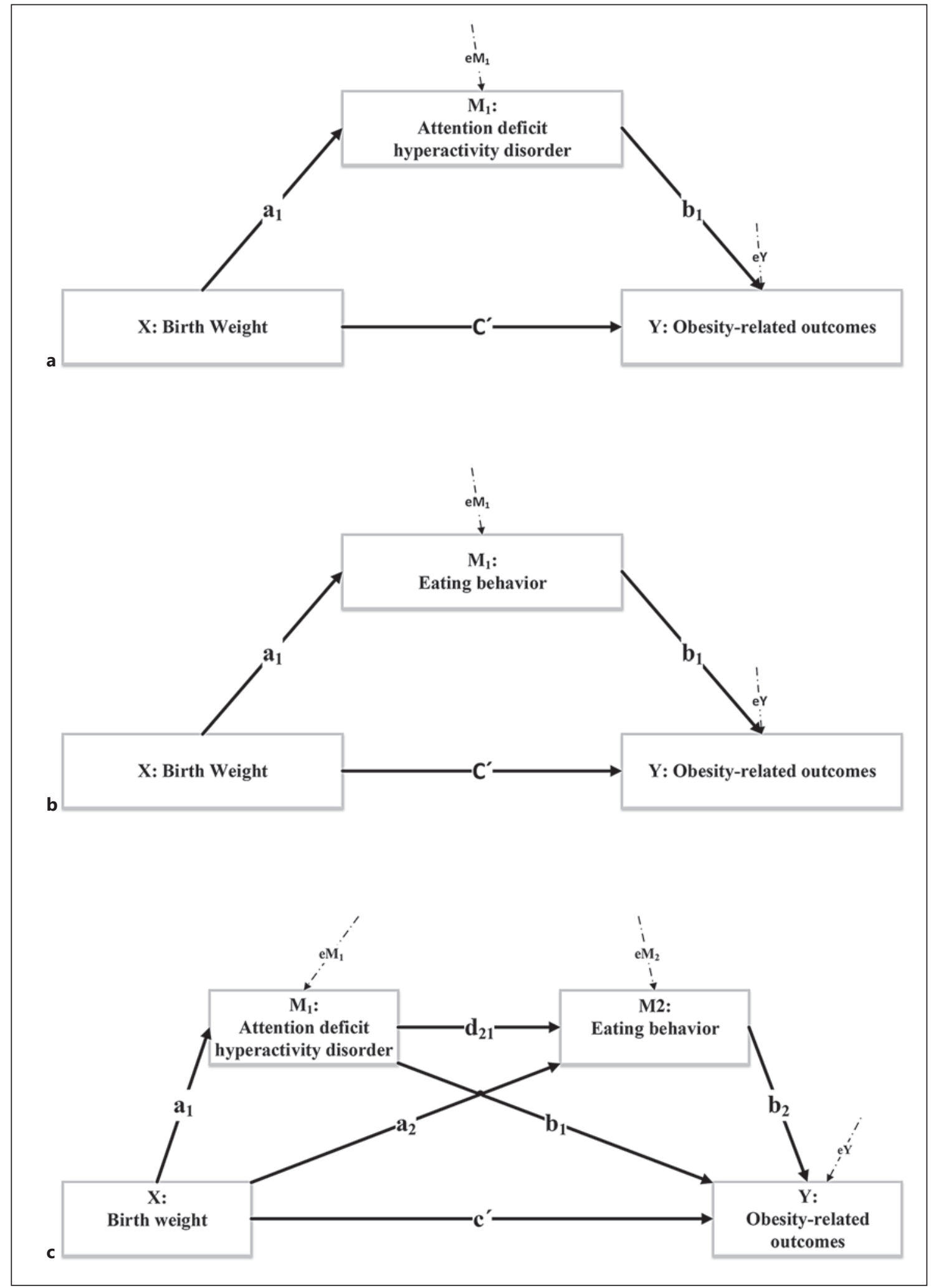

Fig. 1. a Birth weight predicted obesity-related outcomes of children indirectly through ADHD. b Birth weight predicted obesity-related outcomes of children indirectly through eating behaviour. c Birth weight predicted obesity-related outcomes of children indirectly through both ADHD and eating behaviour. 


\section{Materials and Methods}

\section{Population and Study Design}

The study, a retrospective cohort study, was carried out in Shengjing Hospital of China Medical University between 2017 and 2018, and it aimed to investigate factors of children's health, particularly in obesity and related metabolic diseases. Data were collected when the children were 7-9 years old. The critical criterion for inclusion was birth weight of 2,000-2,500 g. However, children born to mothers prior to 34 weeks gestation, with severe complications during pregnancy and congenital neurological diseases, as part of multiple pregnancies (e.g., twins or triplets) or without complete data were excluded. Finally, the cohort consisted of 677 children, of whom 277 were MLBW (2,000-2,499 g), and 450 were normal birth weight (NBW, 2,500-3,999 g). Each LBW child was paired with 2 NBW children of the same sex and age. The weight and length $Z$ scores at birth were calculated based on the World Health Organization (WHO) child growth reference [24].

\section{Measures}

In-person visits were conducted with children of 7-9 years old to record their demographic information and collect anthropometric outcomes and biological samples, which took about $60 \mathrm{~min}$ to complete.

\section{Anthropometric and Biological Outcomes}

All participants had complete physical measurements taken, which included weight, height, WC, hip circumference (HC), skinfold thickness (SF), body fat, and blood pressure (BP). The weight and length of the infants at birth were recorded from the medical records. According to a standard protocol, weight and height at 7-9 years old were measured with a digital scale and a stadiometer while children wore no shoes and light clothes (Seca 861 and 214; Seca Corporation, Hamburg, Germany) by trained interviewers. $Z$-scored body mass indexes (BMIs) for age and sex were calculated based on the WHO child growth reference and classified as not overweight and overweight or obese [25].

WC was measured using the Seca automatic roll-up measuring tape (accurate to $0.1 \mathrm{~cm}$ ) at a level midway between the lowest ribs and the superior iliac crest at the end of normal inhalation/exhalation. $\mathrm{HC}$ was measured at the maximum extension of the buttocks. The average of two consecutive measurements was used for analysis. The waist-to-height ratio was calculated as dividing WC $(\mathrm{cm})$ by height $(\mathrm{cm})$, while the waist-to-hip ratio was calculated as dividing WC by HC. $Z$-scored WCs for age and sex were calculated, and abdominal obesity was defined as WC $Z$ score $>90$ th percentile [26].

The subscapular, triceps, and subumbilical SFs were measured 3 times with callipers and averaged. Bioelectrical impedance analysis was applied to examine body composition (fat mass percentage) by using the TANITA DC-430 MA. This method has been proven to be highly correlated $(r=0.96)$ with dual-energy X-ray absorptiometry scan measurements [27].

$\mathrm{BP}$ was measured by an electronic BP monitor for children and adolescents (OMRON M6; Beijing, China). Participants were advised to avoid caffeinated beverages and physical activity (PA) on the morning of the measurement. Before the measurement, the subjects were told to sit still for $10 \mathrm{~min}$. BP was measured three times, with 5-min intervals. $Z$-scored BP for age, sex, and height were calculated based on "The Fourth Report on the Diagnosis,
Evaluation and Treatment of High Blood Pressure in Children and Adolescents" [28].

Fasting venous blood samples were obtained between 07:00 and 08:30 and measured for lipids (total cholesterol, triglycerides, high-density lipoprotein [HDL] cholesterol, and low-density lipoprotein cholesterol) and fasting blood glucose (FBG). We constructed $Z$ scores for the triglyceride level, HDL cholesterol level, and FBG level of children using sex and age standardization [26].

\section{Attention-Deficit Hyperactivity Disorder}

The emotional and behavioural assessment of children was performed with the Chinese translation of the Parent Symptom Questionnaire by Conners [29]. The scale consists of 48 items that are divided into six dimensions depending on the following characteristics: conduct problems, learning problems, psychosomatic disorder, impulsive, anxiety, and ADHD. This study focused on the dimension of ADHD. The scale was answered by parents on a 4 -point scale $(0,1,2$, and 3$)$. It used $x \pm S D$ to express normal range values, and a child with a clinically concerning ADHD symptom $Z$ score two standard deviations above the mean was considered abnormal. For example, the normal range for ADHD was $0.7 \pm 0.6$, and a score greater than 1.9 indicated that the child had ADHD symptoms [29]. The Cronbach's alpha for this dimension was 0.87.

\section{Eating Behaviour}

The assessment of the eating behaviour of children was measured by the Chinese translation of the Child Eating Behaviour Questionnaire, which contains 35 items and consists of eight subscales (food responsiveness, enjoyment of food, satiety responsiveness, slowness in eating, fussiness, desire to drink, emotional undereating, and emotional overeating) [30]. The scale was answered by the parents on a 5-point Likert scale, which ranged from "never" to "always." The higher score on a dimension reflected more frequent enactment of this child's eating behaviour. These subscales showed high reliability in our study with Cronbach's alpha ranging from 0.80 to 0.95 .

\section{Covariates}

Based on previous research [31-33], other variables were used to describe maternal and children's demographic characteristics and to control confounding variables. These variables were collected from hospital records (maternal age, delivery mode, health status during pregnancy, parity, child's sex, gestational weeks, and physical growth of newborns) and questionnaires (parental BMI, parental education, annual family income, mode of infant feeding, smoking during pregnancy, child's age, childhood screen time, and childhood PA time).

\section{Statistical Analyses}

The data were initially cleaned and analysed to explore the relationships between variables and examine potential confounding factors. Based on the preliminary analysis, the mediating effect was analysed.

\section{Preliminary Analysis}

Descriptive analysis of study samples was stratified by birth weight and expressed as mean (SD) or percentage. The variables from the lipids and blood glucose profiles had a skewed distribution and were log-transformed before analysis [34]. The $\chi^{2}$ test and $T$ test were used for the unilabiate comparative analysis, and we 
Table 1. Obesity-related outcomes and behavioural factors according to birth weight categories

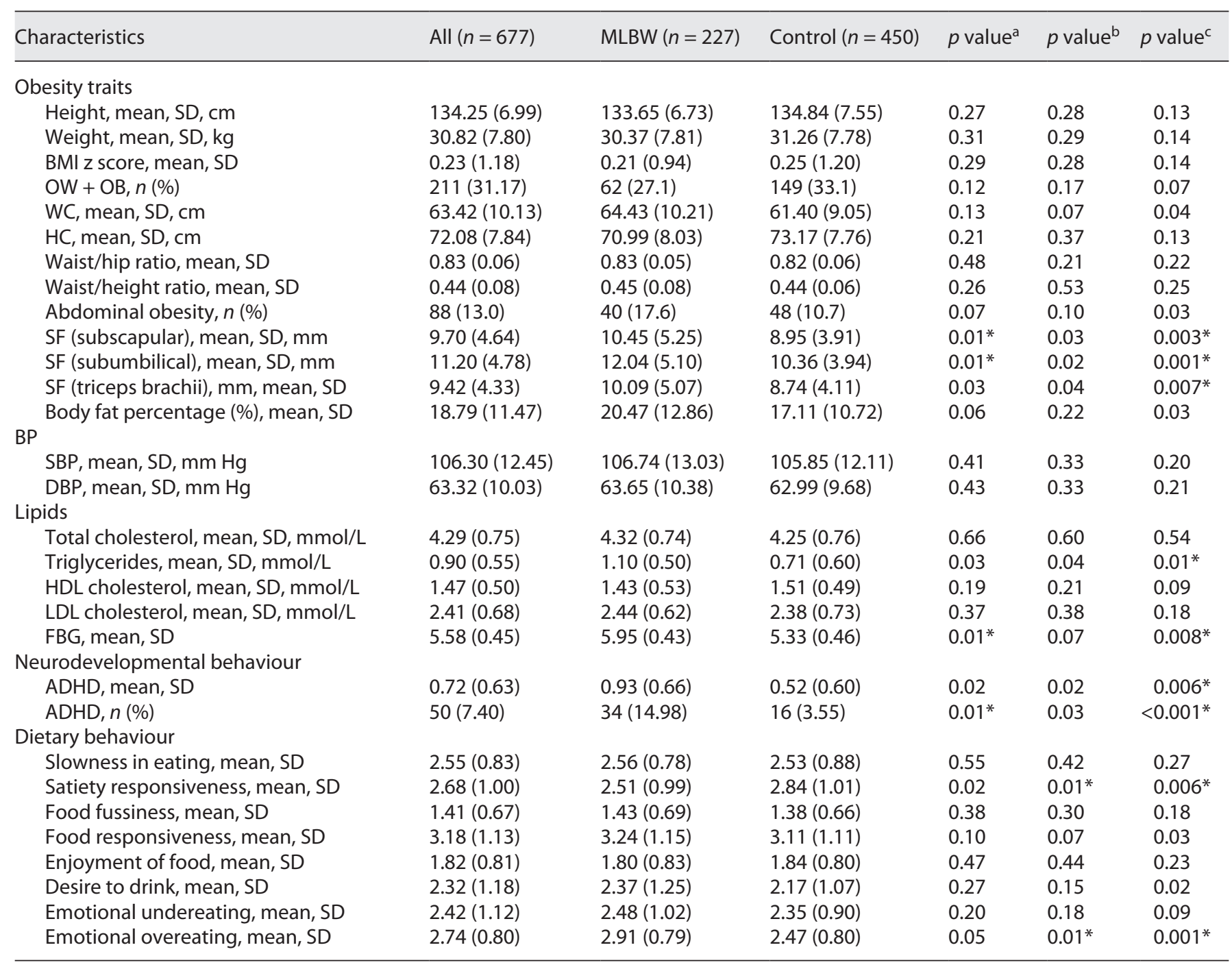

Revised a of the Bonferroni method $=0.05 / 3=0.017$. DBP, diastolic blood pressure; SBP, systolic blood pressure; LDL, low-density lipoprotein; cm, centimetres. ${ }^{*} p<0.017$. ${ }^{\mathrm{a}}$ All versus MLBW. ${ }^{\mathrm{b}}$ All versus control. ${ }^{\mathrm{c}} \mathrm{MLBW}$ versus control.

had handled repeated testing by the Bonferroni method. Partial correlation analysis was used to assess the association between birth weight, ADHD, abnormal eating, and obesity-related behaviour to explore whether there was a significant correlation between the variables to warrant carrying out an analysis of mediating effects.

To examine the relationships between birth weight (NBW and MLBW) and obesity-related outcomes, logistic regression analyses were conducted to analyse the relationships between birth weight (NBW and MLBW) and classified obesity-related outcomes (overweight and obesity and abdominal adiposity). The linear regression analyses were conducted to analyse the relationships between birth weight (NBW and MLBW) and continuous obesity-related outcomes (BMI $Z$ score, WC, waist/hip ratio, waist/height ratio, SF, body fat percentage, diastolic/systolic BP, total cholesterol, triglycerides, low-density lipoprotein cholesterol, HDL cholesterol, and FBG). We performed the above analyses using five models: model 1 analyses were adjusted for sex, age (years), gestation age at birth, breastfeeding duration, PA group, sedentary time, maternal age, parental BMI, parent with a university degree, smoking or passive smoking during pregnancy, gestational diabetes mellitus, hypertension during pregnancy, caesarean section, Apgar score 5 min after birth, and household income; model 2: model $1+$ BMI $Z$ score of children; model 3: model $2+$ ADHD; model 4: model $2+$ satiety responsiveness; and model 5: model $2+$ emotional overeating. The results are shown as adjusted $\beta$ estimates $(a \beta s)$ and adjusted odds ratios with $95 \%$ CIs. In sensitivity analyses, we tested the significance between cohort characteristics and the outcomes of MLBW children and control children stratified by sex. 
Table 2. Logistic regression analysis of MLBW and obesity-related outcomes

\begin{tabular}{cll}
\hline & Control $(n=450)$ & MLBW $(n=227)$ \\
\hline OW + OB & & \\
Model 1 & Ref & $0.72(0.40,1.29)$ \\
Model 2 & Ref & $0.69(0.37,1.28)$ \\
Model 3 & Ref & $0.61(0.32,1.18)$ \\
Model 4 & Ref & $0.66(0.35,1.26)$ \\
Model 5 & Ref & $0.68(0.22,2.09)$ \\
Abdominal adiposity & & \\
Model 1 & Ref & $1.03(0.88,1.43)$ \\
Model 2 & Ref & $1.97(1.04,3.76)^{*}$ \\
Model 3 & Ref & $1.37(0.71,2.63)$ \\
Model 4 & Ref & $1.87(0.92,3.49)$ \\
Model 5 & Ref & $1.33(0.70,2.55)$ \\
\hline
\end{tabular}

Model 1 analysis was adjusted for sex, age (y), gestation age at birth, breastfeeding duration, PA group, sedentary time, maternal age, parental BMI, parent with a university degree, smoking or passive smoking during pregnancy, gestational diabetes mellitus, hypertension during pregnancy, caesarean section, Apgar score 5 min after birth, and household income; model 2: model $1+$ children BMI $Z$ score; model 3: model $2+$ ADHD; model 4: model $2+$ satiety responsiveness; and model 5: model $2+$ emotional overeating. ${ }^{*} p<0.05$

\section{Mediating Effect Analyses}

The analyses of mediating effects were performed using bootstrapping analysis with PROCESS macro for SPSS (IBM Corp. Released 2012. IBM SPSS Statistics for Windows, Version 21.0. Armonk, NY: IBM Corp.) and Mplus version $7.4[35,36]$. The method was designed to evaluate the total effects, direct effects, and mediating effects shown by the regression coefficient and 95\% CI. Firstly, we performed the mediation analysis to test whether the relationship between birth weight and obesity-related outcomes was mediated by ADHD or eating behaviour. Secondly, we performed the mediation analysis to test whether the relationship between birth weight and obesity-related outcomes was mediated by ADHD and eating behaviour gradually. All analyses were adjusted for sex, age (years), gestational age at birth, breastfeeding duration, PA group, sedentary time, maternal age, parental BMI, the parent with a university degree, smoking or passive smoking during pregnancy, gestational diabetes mellitus, hypertension during pregnancy, caesarean section, Apgar score 5 min after birth, household income, and BMI $Z$ score of children.

\section{Results}

\section{Preliminary Analyses}

Descriptive and Bivariate Analyses

The perinatal and demographic characteristics of subjects by birth weight category are shown in online supplementary Table S1 (for all online suppl. material, see www. karger.com/doi/10.1159/000520902), and the anthropo-
Table 3. Linear regression analysis of MLBW and obesity-related outcomes

\begin{tabular}{|c|c|c|}
\hline & Control $(n=450)$ & $\operatorname{MLBW}(n=227)$ \\
\hline \multicolumn{3}{|l|}{ BMI Z score } \\
\hline Model 1 & Ref & $-0.22(-1.09,0.66)$ \\
\hline Model 2 & Ref & $-0.18(-1.03,0.67)$ \\
\hline Model 3 & Ref & $-0.14(-0.67,0.39)$ \\
\hline Model 4 & Ref & $-0.21(-1.07,0.66)$ \\
\hline Model 5 & Ref & $-0.13(-0.60,0.31)$ \\
\hline \multicolumn{3}{|l|}{ WC } \\
\hline Model 1 & Ref & $0.25(-0.10,0.60)$ \\
\hline Model 2 & Ref & $0.60(0.24,0.96)^{*}$ \\
\hline Model 3 & Ref & $0.35(0.07,0.63)^{*}$ \\
\hline Model 4 & Ref & $0.37(0.10,0.54)^{*}$ \\
\hline Model 5 & Ref & $0.32(0.05,0.59)^{*}$ \\
\hline \multicolumn{3}{|c|}{ Waist/hip ratio } \\
\hline Model 1 & Ref & $0.08(-0.19,0.35)$ \\
\hline Model 2 & Ref & $0.07(-0.20,0.34)$ \\
\hline Model 3 & Ref & $0.03(-0.25,0.30)$ \\
\hline Model 4 & Ref & $0.05(-0.22,0.33)$ \\
\hline Model 5 & Ref & $0.09(-0.07,0.26)$ \\
\hline \multicolumn{3}{|c|}{ Waist/height ratio } \\
\hline Model 1 & Ref & $-0.11(-0.33,0.12)$ \\
\hline Model 2 & Ref & $-0.14(-0.37,0.09)$ \\
\hline Model 3 & Ref & $-0.19(-0.42,0.04)$ \\
\hline Model 4 & Ref & $-0.16(-0.39,0.07)$ \\
\hline Model 5 & Ref & $-0.12(-0.35,0.11)$ \\
\hline \multicolumn{3}{|r|}{ 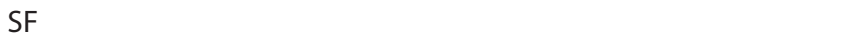 } \\
\hline Model 1 & Ref & $0.63(0.27,1.53)^{*}$ \\
\hline Model 2 & Ref & $0.67(0.31,1.03)^{*}$ \\
\hline Model 3 & Ref & $0.35(0.02,0.69)^{*}$ \\
\hline Model 4 & Ref & $0.60(0.25,0.95)^{*}$ \\
\hline Model 5 & Ref & $0.63(0.35,0.91)^{*}$ \\
\hline \multicolumn{3}{|c|}{ Body fat percentage } \\
\hline Model 1 & Ref & $0.27(-0.03,0.51)$ \\
\hline Model 2 & Ref & $0.64(0.31,0.97)^{*}$ \\
\hline Model 3 & Ref & $0.29(0.03,0.55)^{*}$ \\
\hline Model 4 & Ref & $0.53(0.21,0.85)^{*}$ \\
\hline Model 5 & Ref & $0.28(0.07,0.49)^{*}$ \\
\hline
\end{tabular}

Model 1 analysis was adjusted for sex, age (y), gestation age at birth, breastfeeding duration, PA group, sedentary time and feeling system disorder, maternal age, parental BMI, parent with a university degree, smoking or passive smoking during pregnancy, gestational diabetes mellitus, hypertension during pregnancy, caesarean section, Apgar score 5 min after birth, and household income; model 2: model 1 + children BMI Z score; model 3: model 2 + ADHD; model 4: model $2+$ satiety responsiveness; and model 5 : model $2+$ emotional overeating. ${ }^{*} p<0.05$.

metric parameters and behavioural factors by birth weight category are shown in Table 1. Compared with the NBW group, the MLBW group had a higher proportion of caesarean sections. In terms of the newborn physical indicators, the MLBW group had a significantly lower number 
than that in the NBW group. In addition to household income, no significant differences in demographic characteristics were found between the 2 groups.

However, most anthropometric parameters and behavioural factors at the age of 7-9 years were significantly different between the MLBW and NBW groups. Based on the above results, correlations between birth weight, $\mathrm{ADHD}$, eating behaviour, and obesity-related outcomes after adjusting for gender are indicated in online supplementary Table S2. We found that there were significant correlations among birth weight, $\mathrm{ADHD}$, satiety responsiveness, emotional overeating, and partial obesity indexes (WC, SF, body fat percentage, triglycerides, and FBG). In sensitivity analyses, the analyses stratified by sex yielded similar results and are shown in online supplementary Tables S3 and S4.

\section{Analyses of Regression}

As shown in Tables 2-4, the relationships between children's birth weight and obesity-related outcomes were performed. Before adding the BMI $Z$ score of children to models, the children with MLBW tended to have higher levels of SF, triglycerides, and FBG. However, after further adjusting for the current BMI $Z$ score of children, except for the above results, lower birth weight was related to higher WC ( $\mathrm{a} \beta=0.60,95 \% \mathrm{CI}$ : $0.24-0.96)$, body fat percentage $(\mathrm{a} \beta=0.64,95 \% \mathrm{CI}: 0.31-0.97)$, and risk of abdominal obesity (adjusted odds ratio $=1.97,95 \% \mathrm{CI}$ : 1.04-3.76). Furthermore, after adjustment for ADHD (model 3), satiety responsiveness (model 4 ), and emotional overeating (model 5) one by one, most of the relationships either were attenuated but remained or became non-significant, which suggested the potential mediating role of ADHD, satiety responsiveness, or emotional overeating. Because other factors did not affect the relationship between birth weight and obesity, they were not shown in the study.

\section{Mediation Analyses}

We examined two models, the first of which explored how many behaviour factors (ADHD or eating behaviour) contributed to the associations between birth weight and obesity-related outcomes. In Figure 2a, modelling the relationship between variables indicates that the increased risk of ADHD mediates $23.0 \%$ of the $\mathrm{BW}-\mathrm{WC}$ association. In the first equation, $\mathrm{BW}$ was negatively related to $\mathrm{ADHD}(\beta=-0.45, p<0.05)$. In the second equation, ADHD was positively correlated with $\mathrm{WC}(\beta=0.31, p<0.05)$. In the third equation, $\mathrm{BW}$ was negatively associated with WC $(\beta=-0.35, p<0.05)$. Fi-
Table 4. Linear regression analysis of MLBW and metabolismrelated outcomes

\begin{tabular}{|c|c|c|}
\hline & Control $(n=450)$ & $\operatorname{MLBW}(n=227)$ \\
\hline \multicolumn{3}{|l|}{ Diastolic BP } \\
\hline Model 1 & Ref & $-0.11(-0.38,0.17)$ \\
\hline Model 2 & Ref & $-0.09(-0.37,0.18)$ \\
\hline Model 3 & Ref & $-0.09(-0.37,0.19)$ \\
\hline Model 4 & Ref & $-0.06(-0.31,0.20)$ \\
\hline Model 5 & Ref & $-0.07(-0.34,0.20)$ \\
\hline \multicolumn{3}{|l|}{ Systolic BP } \\
\hline Model 1 & Ref & $0.04(-0.23,0.32)$ \\
\hline Model 2 & Ref & $0.03(-0.24,0.31)$ \\
\hline Model 3 & Ref & $0.02(-0.26,0.30)$ \\
\hline Model 4 & Ref & $0.01(-0.28,0.29)$ \\
\hline Model 5 & Ref & $0.03(-0.25,0.31)$ \\
\hline \multicolumn{3}{|c|}{ Total cholesterol } \\
\hline Model 1 & Ref & $0.05(-0.22,0.33)$ \\
\hline Model 2 & Ref & $0.03(-0.25,0.31)$ \\
\hline Model 3 & Ref & $0.01(-0.27,0.30)$ \\
\hline Model 4 & Ref & $0.02(-0.26,0.30)$ \\
\hline Model 5 & Ref & $0.04(-0.11,0.11)$ \\
\hline \multicolumn{3}{|l|}{ Triglycerides } \\
\hline Model 1 & Ref & $0.47(0.15,0.79)^{*}$ \\
\hline Model 2 & Ref & $0.40(0.10,0.70)^{*}$ \\
\hline Model 3 & Ref & $0.34(0.10,0.58)^{*}$ \\
\hline Model 4 & Ref & $0.42(0.20,0.64)^{*}$ \\
\hline Model 5 & Ref & $0.41(0.12,0.94)^{*}$ \\
\hline \multicolumn{3}{|c|}{ LDL cholesterol } \\
\hline Model 1 & Ref & $0.03(-0.24,0.30)$ \\
\hline Model 2 & Ref & $0.02(-0.26,0.29)$ \\
\hline Model 3 & Ref & $0.01(-0.27,0.29)$ \\
\hline Model 4 & Ref & $0.03(-0.26,0.31)$ \\
\hline Model 5 & Ref & $0.02(-0.26,0.29)$ \\
\hline \multicolumn{3}{|c|}{ HDL cholesterol } \\
\hline Model 1 & Ref & $-0.19(-0.50,0.11)$ \\
\hline Model 2 & Ref & $-0.15(-0.44,0.14)$ \\
\hline Model 3 & Ref & $-0.17(-0.47,0.13)$ \\
\hline Model 4 & Ref & $-0.18(-0.49,0.12)$ \\
\hline Model 5 & Ref & $-0.12(-0.50,0.25)$ \\
\hline \multicolumn{3}{|l|}{ FBG } \\
\hline Model 1 & Ref & $0.42(0.11,0.73)^{*}$ \\
\hline Model 2 & Ref & $0.38(0.08,0.68)^{*}$ \\
\hline Model 3 & Ref & $0.30(0.02,0.58)^{*}$ \\
\hline Model 4 & Ref & $0.46(0.17,0.69)^{*}$ \\
\hline Model 5 & Ref & $0.40(0.12,0.68)^{*}$ \\
\hline
\end{tabular}

Model 1 analysis was adjusted for sex, age (y), gestation age at birth, breastfeeding duration, PA group, sedentary time and feeling system disorder, maternal age, parental BMI, parent with a university degree, smoking or passive smoking during pregnancy, gestational diabetes mellitus, hypertension during pregnancy, caesarean section, Apgar score 5 min after birth, and household income; model 2: model 1 + children BMI Z score; model 3: model 2 + ADHD; model 4: model $2+$ satiety responsiveness; and model 5 : model $2+$ emotional overeating. LDL, low-density lipoprotein. ${ }^{*} p<0.05$. 


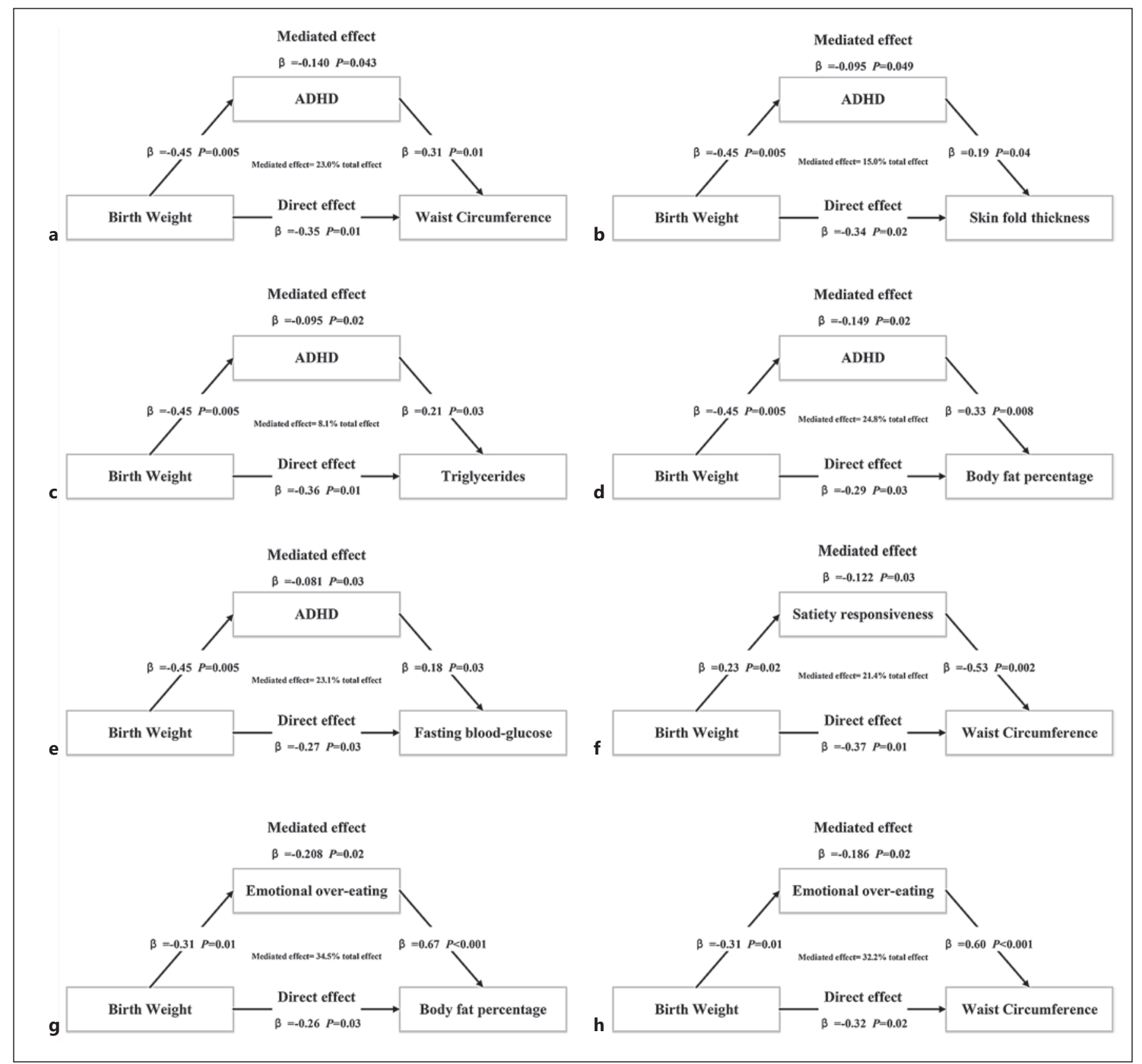

Fig. 2. a-e Models: ADHD mediation models of the associations between birth weight and obesity-related outcomes in children. f-h Models: eating behaviour mediation models of the associations between birth weight and obesity-related outcomes in children. All analyses are adjusted for sex, age (years), gestational age at birth,

nally, the results showed that the negative correlation of BW and WC was mediated by ADHD. Figure $2 \mathrm{~b}-\mathrm{h}$ suggest similar mediation models for the relationships of birth weight with SF, triglycerides, body fat percentage, and FBG.

breastfeeding duration, PA group, sedentary time, maternal age, parental BMI, parent with a university degree, smoking during pregnancy, health status during pregnancy, caesarean section, Apgar score 5 min after birth, household income, and the BMI $Z$ score of children.

The second aim was that BW predicted obesity-related outcomes indirectly via the abnormal eating behaviour of children caused by ADHD (Table 5). We did discover support for this aim that lower birth weight predicted higher WC indirectly through emotional overeating 
Table 5. Indirect effects of birth weight, ADHD, and eating behaviour on child obesity-related outcomes ${ }^{\mathrm{a}}$

\begin{tabular}{lll}
\hline & $\beta$ & $95 \% \mathrm{Cl}$ \\
\hline Total effect & -0.56 & $(-0.79,-0.19)$ \\
Direct effect & -0.27 & $(-0.39,-0.15)$ \\
Indirect effect & & \\
$\quad(X)$ Birth weight $\rightarrow(\mathrm{M} 1)$ ADHD $\rightarrow(\mathrm{Y})$ WC & -0.14 & $(-0.25,-0.04)$ \\
$(\mathrm{X})$ Birth weight $\rightarrow(\mathrm{M} 2)$ emotional overeating $\rightarrow(\mathrm{Y})$ WC & -0.19 & $(-0.29,-0.10)$ \\
$(\mathrm{X})$ Birth weight $\rightarrow(\mathrm{M} 1)$ ADHD $\rightarrow(\mathrm{M} 2)$ emotional overeating & -0.15 & $(-0.21,-0.09)$ \\
$(\mathrm{M} 1)$ ADHD $\rightarrow(\mathrm{M} 2)$ emotional overeating $\rightarrow(\mathrm{Y})$ WC & 0.21 & $(0.12,0.32)$ \\
$(\mathrm{X})$ Birth weight $\rightarrow(\mathrm{M} 1)$ ADHD $\rightarrow(\mathrm{M} 2)$ emotional overeating $\rightarrow(\mathrm{Y})$ WC & -0.10 & $(-0.19,-0.01)$ \\
\hline
\end{tabular}

${ }^{a}$ All models adjusted for sex, age (y), gestation age at birth, breastfeeding duration, PA group, sedentary time; maternal age, parental BMI, parent with a university degree, smoking or passive smoking during pregnancy, gestational diabetes mellitus, hypertension during pregnancy, caesarean section, Apgar score 5 min after birth, household income, and children BMI Z score.

caused by ADHD, independently of the BMI $Z$ score of children and other confounding factors. No other mediating analyses were significant, and they were not shown in the study.

\section{Discussion/Conclusion}

The preliminary prediction of our study was that birth weight would be negatively related to obesity-related outcomes, and the associations were mediated, partially, by an increased risk of ADHD or abnormal eating behaviour after being adjusted for the BMI $Z$ score, thus describing a mechanism whereby acquired behaviour problems might be highlighting the MLBW-OB relationship. Furthermore, our study elucidates that birth weight was inversely related to $\mathrm{ADHD}$ and then abnormal eating, which in turn predicted WC in children, so the roles of ADHD and eating behaviour, especially in emotional overeating, were affected in the MLBW-WC relationship gradually.

The results of our study were in line with previous research suggesting that children with LBW tend to have trouble with obesity-related outcomes. Firstly, Yuan and colleagues investigated the role of birth weight on general and central obesity in 16,580 Chinese children and adolescents and found that children with LBW had higher risk of central obesity (OR: 2.30; 95\% CI: 1.03-5.14) [37], and the results were also supported by the Exploring Perinatal Outcomes among Children Study [3]. Secondly, LBW children were found to have higher than average FBG levels, which is similar to the Japan Nurses' Health Study [38]. Thirdly, our study provided evidence strik- ingly similar to that from Brian and colleagues who explored the relationships between LBW and lipids in 575 adolescents aged $14-18$ years and found a significant negative association between birth weight and triglycerides [39]. Fourthly, a study in the Southampton region of England indicated that a decrease in BW of 1 SD was related with an increase of about $2 \%$ in fat mass, which was also consistent with our results [40]. However, the existing studies on LBW, especially on MLBW, and obesity-related outcomes are still inconsistent. Two cohort studies detected that there were no significant differences in obesity-related outcomes in LBW or MLBW participants compared with controls $[8,12]$. On the other hand, the studies executed in China and Finland had found an association between $\mathrm{BP}$ and BMI with LBW $[4,5]$.

Previously, research has suggested that these alterations in obesity-related outcomes were caused by undesirable foetal programming and not just by adiposity. However, we further found an indirect LBW-OB relationship through ADHD and/or abnormal eating. The mechanisms underlying the mediating role of ADHD symptoms or eating behaviour in the relationships between birth weight and obesity-related outcomes are not well understood. Few studies have hypothesized that nutritional deprivation in utero leads to impaired neurodevelopment and effects on food preferences and later intake patterns, which is reflected in abnormal eating and ADHD $[14,19]$.

In terms of $\mathrm{ADHD}$, our mediation analysis further revealed that increased ADHD symptoms mediated 23.0\%, $15.0 \%, 8.1 \%, 24.8 \%$, and $23.1 \%$ of the risk effect of LBW against WC, SF, triglycerides, body fat percentage, and FBG, respectively (Fig. 2a-e). Cohen and colleagues sug- 
gested that the mediated effects can be divided into three classes according to clinical criteria. If the mediating effect is between $20 \%$ and $80 \%$, it is a partial mediating effect. If the mediating effect is less than $20 \%$, there is no mediating effect, and if the mediating effecting is more than $80 \%$, there is a full mediating effect [41]. In our study, most mediated effects were more than $20 \%$, so the results would have certain important clinical significance. Furthermore, we could not confirm a significant mediatory role for $\mathrm{ADHD}$ in the $\mathrm{BW}-\mathrm{BMI}$ relationship. The findings are similar to those of Olivia et al. [42], who, in 2018 , in a sample of participants who were 10 years of age, found that the relationship between birth weight and BMI was not mediated by ADHD. Although the mechanism linking birth weight and other obesity-related outcomes is not fully elucidated, it has been suggested that ADHD is the crucial factor in these relationships. We did observe that hypoxaemia during pregnancy is not the only factor that may lead to LBW, but it also produces lifelong adverse effects on neurodevelopmental function, which in turn increases the risk of ADHD. In addition to birth weight, different growth environments may promote the ability of children to adapt to different states, with subsequent influences on body indexes [43-45].

With regard to abnormal eating behaviour, our mediation analysis further revealed that increased satiety responsiveness and emotional overeating mediated $21.4 \%$ and $32.2 \%$ of the risk effect of LBW against WC, respectively (Fig. 2f-h). According to Cohen's proposed theory [41], the clinical value of these mediated effects could be recognized. Our findings were in line with previous studies. Derks et al. [46] found the relationship between birth weight and eating behaviour and suggested that the development and maintenance of unhealthy eating behaviours were potential correlates of the higher weight status. Moreover, our findings were also concordant with the results of the Generation XXI birth cohort study, which showed that adherence at 7 years of age to a dietary pattern rich in energy-dense foods may increase several cardiometabolic parameters at 10 years of age [47]. Although the results of previous studies were consistent with parts of our results, the research on the mediation analysis and its mechanisms are still rather sparse. We did observe that it may be similar to the mechanism of ADHD. On one hand, intrauterine growth restriction programmes both the satiety and appetite mechanisms and on the other hand, parents of LBW children, especially those with MLBW, would be more likely to overfeed or adopt a motivation mechanism to promote the development of their children and might easily slip into poor eating habits, such as emotional eating, which may ultimately cause obesity and metabolic diseases [18, 48, 49].

Intriguingly, in our study, the roles of ADHD and emotional overeating were affected by the MLBW-WC relationship gradually in a model. It may be because of the similarity of the mechanisms in the BW-ADHD-OB and $\mathrm{BW}-\mathrm{OE}-\mathrm{BO}$ relationships, and the study by Van pointed out that ADHD may contribute to obesity and other related outcomes through disordered eating patterns [19]. However, current evidence is limited and needs to be explored further.

To date, few studies have used a bootstrapping approach to analyse the relationships between birth weight and obesity-related outcomes. To the best of our knowledge, our research is the first to explore the mediating role of both ADHD and abnormal eating in the association of MLBW and obesity-related outcomes in a cohort of Chinese children. In addition, the current study adjusted for comprehensive potential confounding factors, including demographic information on the mother, child, and family; lifestyle factors; and BMI $Z$ score of children, so it could accurately examine the relationships between birth weight with obesity and its subsequent complications. Although our study presents novelty in the field of prevention of obesity and metabolic diseases in children, there were also several limitations, of which an acknowledgement could provide constructive recommendations for future research. Firstly, our study was limited in its retrospective cohort nature, and recall bias may potentially impact on our data. Secondly, the questionnaire was answered by the mother, so we could not eliminate social desirability bias completely. Thirdly, while the Parent Symptom Questionnaire of Conners is a global authoritative tool to examine behaviour problems in children, it may have limited capacity for the diagnosis of ADHD and other related problems accurately and systematically. Fourthly, our study included a relatively high number of tests for significance. In sensitivity analyses however the results did not change following adjustment for sex. A more comprehensive and careful scale of participants should be executed in further research to solve this limitation.

In conclusion, our study demonstrated that the efficacy of both ADHD and eating behaviour should be considered when assessing risk for birth weight and childhood obesity-related outcomes. Interventions modifying $\mathrm{ADHD}$ and abnormal eating suggest promise for reducing the risk of obesity and metabolic diseases caused by LBW, especially MLBW. Furthermore, an extended investigation of a prospective study in young children is required to explore whether the associations are consis- 
tent in later life. These recommendations are essential to prove the cause and effect and develop effective intervention strategies to prevent childhood obesity-related outcomes. Interventions stemming from our study might provide a novel and promising way to help parents to focus on behaviour problems in children to create a delicate growth environment while promoting the healthy development of children both physically and mentally.

\section{Acknowledgments}

We are grateful to all the participating families who took part in our study. All authors thank the operation team and study team, which include interviewers, nurses, laboratory technicians, research scientists, and volunteers.

\section{Statement of Ethics}

All procedures were approved by the Medical Ethics Committee of China Medical University (application reference 71774173). The research was conducted ethically in accordance with the World Medical Association Declaration of Helsinki. The parents were informed of the project requirements and were provided with informed consent forms and signed them prior to enrolment.

\section{Conflict of Interest Statement}

The authors declare no conflict of interest. The funders had no role in the design of the study; in the collection, analysis, or interpretation of data; in the writing of the manuscript; or in the decision to publish the results.

\section{Funding Sources}

The study was supported by the China Postdoctoral Science Foundation (2019M661179).

\section{Author Contributions}

Conceptualization: X.T.W. and Y.N.M.; methodology: X.T.W. and Y.L.; software: X.T.W.; validation: J.J.H. and Y.N.M.; formal analysis: X.T.W.; investigation: X.T.W. and J.J.H.; resources Y.L. and D.L.W.; data curation: D.L.W.; writing - original draft preparation: X.T.W.; writing - review and editing: J.J.H. and Y.N.M.; project administration: Y.N.M.; and funding acquisition: D.L.W. All the authors have read and agreed to the published version of the manuscript.

\section{References}

1 NCD Risk Factor Collaboration (NCD-RisC). Worldwide trends in body-mass index, underweight, overweight, and obesity from 1975 to 2016: a pooled analysis of 2416 populationbased measurement studies in 128.9 million children, adolescents, and adults. Lancet. 2017;390(10113):2627-42.

2 Lobstein T, Jackson-Leach R, Moodie ML, Hall KD, Gortmaker SL, Swinburn BA, et al. Child and adolescent obesity: part of a bigger picture. Lancet. 2015;385(9986):251020.

3 Jaiswal M, Crume T, Vehik K, Scherzinger A, Stamm E, Hamman RF, et al. Is low birth weight associated with adiposity in contemporary US youth? the exploring perinatal outcomes among children (EPOCH) study. J Dev Orig Health Dis. 2012;3(3): 166-72.

4 Qiao Y, Ma J, Wang Y, Li W, Katzmarzyk PT, Chaput JP, et al. Birth weight and childhood obesity: a 12-country study. Int J Obes Suppl. 2015;5(Suppl 2):S74-9.

5 Hovi P, Vohr B, Ment LR, Doyle LW, McGarvey $\mathrm{L}$, Morrison KM, et al. Blood pressure in young adults born at very low birth weight: adults born preterm international collaboration. Hypertension. 2016;68(4):880-7.

6 Barker DJ. Fetal origins of coronary heart disease. BMJ. 1995;311(6998):171-4.
7 De Boo HA, Harding JE. The developmental origins of adult disease (barker) hypothesis. Aust N Z J Obstet Gynaecol. 2006;46(1):4-14.

8 Darlow BA, Martin J, Horwood LJ. Metabolic syndrome in very low birth weight young adults and controls: the New Zealand 1986 VLBW Study. J Pediatr. 2019;206:128-e5.

9 Ansari H, Qorbani M, Rezaei F, Djalalinia S, Asadi M, Miranzadeh S, et al. Association of birth weight with abdominal obesity and weight disorders in children and adolescents: the weight disorder survey of the CASPIAN-IV Study. J Cardiovasc Thorac Res. 2017;9(3):140-6.

10 Domínguez Hernández C, Klünder Klünder M, Huang F, Flores Armas EM, VelázquezLópez L, Medina-Bravo P. Association between abdominal fat distribution, adipocytokines and metabolic alterations in obese lowbirth-weight children. Pediatr Obes. 2016; 11(4):285-91.

11 Yu ZB, Han SP, Zhu GZ, Zhu C, Wang XJ, Cao XG, et al. Birth weight and subsequent risk of obesity: a systematic review and metaanalysis. Obes Rev. 2011;12(7):525-42.

12 Lindberg J, Norman M, Westrup B, Öhrman T, Domellöf M, Berglund SK. Overweight, obesity, and body composition in 3.5 and 7 -year-old Swedish children born with marginally low birth weight. J Pediatr. 2015; 167(6):1246-e3.
13 Franz AP, Bolat GU, Bolat H, Matijasevich A, Santos IS, Silveira RC, et al. Attention-deficit/ hyperactivity disorder and very preterm/very low birth weight: a meta-analysis. Pediatrics. 2018;141(1):e20171645.

14 van Mil NH, Steegers-Theunissen RP, Motazedi E, Jansen PW, Jaddoe VW, Steegers EA, et al. Low and high birth weight and the risk of child attention problems. J Pediatr. 2015; 166(4):862-9.e1-3.

15 Hanć T, Cortese S. Attention deficit/hyperactivity-disorder and obesity: a review and model of current hypotheses explaining their comorbidity. Neurosci Biobehav Rev. 2018; 92:16-28.

16 Hanć T, Słopień A, Wolańczyk T, DmitrzakWęglarz, Szwed A, Czapla Z, et al. ADHD and overweight in boys: cross-sectional study with birth weight as a controlled factor. Eur Child Adolesc Psychiatry. 2014;24(1):41-53.

17 Quesada D, Ahmed NU, Fennie KP, Gollub EL, Ibrahimou B. A review: associations between attention-deficit/hyperactivity disorder, physical activity, medication use, eating behaviors and obesity in children and adolescents. Arch Psychiatr Nurs. 2018;32(3):495-504.

18 Oliveira A, de Lauzon-Guillain B, Jones L, Emmett P, Moreira P, Ramos E, et al. Birth weight and eating behaviors of young children. J Pediatr. 2015;166(1):59-65. 
19 van Deutekom AW, Chinapaw MJ, Vrijkotte TG, Gemke RJ. The association of birth weight and postnatal growth with energy intake and eating behavior at 5 years of age - a birth cohort study. Int J Behav Nutr Phys Act. 2016;13:15.

20 Nigg JT, Johnstone JM, Musser ED, Long HG, Willoughby MT, Shannon J. Attention-deficit/hyperactivity disorder (ADHD) and being overweight/obesity: new data and meta-analysis. Clin Psychol Rev. 2016;43:67-79.

21 Reinblatt SP, Mahone EM, Tanofsky-Kraff M, Lee-Winn AE, Yenokyan G, Leoutsakos J-MS, et al. Pediatric loss of control eating syndrome: association with attention-deficit/ hyperactivity disorder and impulsivity. Int $J$ Eat Dis. 2015;48(6):580-8.

22 Preacher KJ, Hayes AF. Asymptotic and resampling strategies for assessing and comparing indirect effects in multiple mediator models. Behav Res Methods. 2008;40(3):879-91.

23 Baron RM, Kenny DA. The moderator-mediator variable distinction in social psychological research: conceptual, strategic, and statistical considerations. J Pers Soc Psychol. 1986; 51(6):1173-82.

24 WHO Multicentre Growth Reference Study Group. WHO child growth standards based on length/height, weight and age. Acta Paediatr Suppl. 2006;450:76-85.

25 de Onis M, Lobstein T. Defining obesity risk status in the general childhood population: which cut-offs should we use? Int J Pediatr Obes. 2010;5(6):458-60.

26 Ahrens W, Moreno LA, Mårild S, Molnár D, Siani A, De Henauw S, et al. Metabolic syndrome in young children: definitions and results of the IDEFICS study. Int J Obes. 2014; 38(Suppl 2):S4-14.

27 Ramel A, Geirsdottir OG, Arnarson A, Thorsdottir I. Regional and total body bioelectrical impedance analysis compared with DXA in Icelandic elderly. Eur J Clin Nutr. 2011;65(8): 978-83.

28 National high blood pressure education program working group on high blood pressure in children and adolescents. The fourth report on the diagnosis, evaluation, and treatment of high blood pressure in children and adolescents. Pediatrics. 2004;114(2 Suppl 4th Report):555-76
29 Conners CK. Conners' rating scale manual. New York: North TonawandaMulti-Health Systems, Inc.; 1990.

30 Wardle J, Guthrie CA, Sanderson S, Rapoport L. Development of the children's eating behaviour questionnaire. J Child Psychol Psychiatry. 2001;42(7):963-70.

31 González-Jiménez E, Montero-Alonso MA, Schmidt-RioValle J, García-García CJ, Padez C. Metabolic syndrome in Spanish adolescents and its association with birth weight, breastfeeding duration, maternal smoking, and maternal obesity: a cross-sectional study. Eur J Nutr. 2015;54(4):589-97.

32 Hanć T, Szwed A, Słopień A, Wolańczyk T, Dmitrzak-Węglarz M, Ratajczak J. Perinatal risk factors and ADHD in children and adolescents: a hierarchical structure of disorder predictors. J Attent Disord. 2018;22(9):85563.

33 Kinge JM. Variation in the relationship between birth weight and subsequent obesity by household income. Health Econ Rev. 2017; $7(1): 18$.

34 Stratakis N, Conti DV, Borras E, Sabido E, Roumeliotaki T, Papadopoulou E, et al. Association of fish consumption and mercury exposure during pregnancy with metabolic health and inflammatory biomarkers in children. JAMA Netw Open. 2020;3(3):e201007.

35 Muthèn, Muthèn: Mplus 7.4. 2015.

36 IBM SPSS. IBM Analytics. Available from: http: //www.ibm.com/analytics/us/en/technology/spss/ Accessed 2016 May 13.

37 Yuan ZP, Yang M, Liang L, Fu JF, Xiong F, Liu GL, et al. Possible role of birth weight on general and central obesity in Chinese children and adolescents: a cross-sectional study. Ann Epidemiol. 2015;25(10):748-52.

38 Katanoda K, Noda M, Goto A, Mizunuma H, Lee JS, Hayashi K. Impact of birth weight on adult-onset diabetes mellitus in relation to current body mass index: the Japan Nurses' Health Study. J Epidemiol. 2017;27(9):42834.

39 Stansfield BK, Fain ME, Bhatia J, Gutin B, Nguyen JT, Pollock NK. Nonlinear relationship between birth weight and visceral fat in adolescents. J Pediatr. 2016;174:185-92.
40 Elia M, Betts P, Jackson DM, Mulligan J. Fetal programming of body dimensions and percentage body fat measured in prepubertal children with a 4-component model of body composition, dual-energy X-ray absorptiometry, deuterium dilution, densitometry, and skinfold thicknesses. Am J Clin Nutr. 2007; 86(3):618-24.

41 Cohen J. Statistical power analysis for the behavioral sciences. New York: Academic Press; 1969. p. 490.

42 Linthavong O, O'Shea TM, Allred E, Perrin E, Bauserman M, Joseph RM, et al. Neurocognitive and health correlates of overweight and obesity among ten-year-old children born extremely preterm. J Pediatr. 2018;200:84-90. e4.

43 West-Eberhard MJ. Phenotypic plasticity and the origins of diversity. Annu Rev Ecol Syst. 1989;20(1):249-78

44 Lim KX, Liu CY, Schoeler T, Cecil CAM, Barker ED, Viding E, et al. The role of birth weight on the causal pathway to child and adolescent ADHD symptomatology: a population-based twin differences longitudinal design. J Child Psychol Psychiatry. 2018;59(10): 1036-43.

45 Landau Z, Pinhas-Hamiel O. Attention deficit/hyperactivity, the metabolic syndrome, and type 2 diabetes. Curr Diab Rep. 2019; 19(8):46.

46 Derks IPM, Bolhuis K, Sijbrands EJG, Gaillard R, Hillegers MHJ, Jansen PW. Predictors and patterns of eating behaviors across childhood: results from the Generation R study. Appetite. 2019;141:104295.

47 Pinto A, Santos AC, Lopes C, Oliveira A. Dietary patterns at 7 year-old and their association with cardiometabolic health at 10 yearold. Clin Nutr. 2019;39(4):1195-202.

48 Bischoff AR, Portella AK, Paquet C, Dalle Molle R, Faber A, Arora N, et al. Low birth weight is associated with increased fat intake in school-aged boys. Br J Nutr. 2018;119(11): 1295-302.

49 Derks IPM, Sijbrands EJG, Wake M, Qureshi F, van der Ende J, Hillegers MHJ, et al. Eating behavior and body composition across childhood: a prospective cohort study. Int J Behav Nutr Phys Act. 2018;15(1):96. 